\title{
New Applications of X-Ray Standing-Wave Fields to Solid State Physics
}

\section{Citation}

Andersen, S. Kjaer, J. A. Golovchenko, and G. Mair. 1976. New Applications of X-Ray Standing-

Wave Fields to Solid State Physics. Physical Review Letters 37, no. 17: 1141-1145. doi:10.1103/ physrevlett.37.1141.

\section{Published Version}

doi:10.1103/PhysRevLett.37.1141

\section{Permanent link}

http://nrs.harvard.edu/urn-3:HUL.InstRepos:29407068

\section{Terms of Use}

This article was downloaded from Harvard University's DASH repository, and is made available under the terms and conditions applicable to Other Posted Material, as set forth at http:// nrs.harvard.edu/urn-3:HUL.InstRepos:dash.current.terms-of-use\#LAA

\section{Share Your Story}

The Harvard community has made this article openly available.

Please share how this access benefits you. Submit a story.

Accessibility 
The authors gratefully acknowledge the ass is tance of D. J. Bishop and M. Levy with the design and running of the experiment. We wish to thank D. M. Lee for his continual interest and encouragement and A. L. Fetter for stimulating correspondence.

*Work supported by the National Science Foundation through Grants No. DMR75-08624 and No. DMR72-03210A01, and by the National Science Foundation through the Cornell Materials Science Center, Grant No. DMR7203029, and Materials Science Center Report No. 2713.

${ }^{1}$ D. D. Osheroff, R. C. Richardson, and D. M. Lee, Phys. Rev. Lett. 28, 885 (1972).

${ }^{2}$ T. A. Alvesalo, Yu. D. Anufriyev, H. K. Collan, O. V. Lounasmaa, and P. Wennerström, Phys. Rev. Lett. 30 , 962 (1973); T. A. Alvesalo, H. K. Collan, M. T. Loponen, and M. C. Veuro, Phys. Rev. Lett. 32, 981 (1974); T. A. Alvesalo, H. K. Collan, M. T. Loponen, O. V. Lounasmaa, and M. C. Veuro, J. Low Temp. Phys. 19, 1 (1974).

${ }^{3}$ H. Kojima, D. N. Paulson, and J. C. Wheatley, Phys. Rev. Lett. 32, 141 (1974), and J. Low Temp. Phys. 21. 283 (1975).

${ }^{4}$ A. W. Yanof and J. D. Reppy, Phys. Rev. Lett. $\underline{33}$, 631,1030 (E) (1974).

${ }^{5}$ P. C. Main, C. W. Kiewiet, W. T. Band, J. R. Hook, D. J. Sandiford, and H. E. Hall, J. Phys. C $\underline{9}$, L397 (1976).

${ }^{6}$ E. L. Andronikashvili, Zh. Eksp. Teor. Fiz. 16, 780 (1946).
${ }^{7}$ For a comprehensive review of the subject see the articles by A. J. Leggett, Rev. Mod. Phys. 47, 331 (1975), and J. C. Wheatley, Rev. Mod. Phys. 47, 415 (1975).

${ }^{8}$ The NMR results along with a complete description of the apparatus will be published shortly. Data were taken as the temperature of the apparatus drifted at rates of $50-500 \mu \mathrm{K} / \mathrm{h}$. A brief description of the cooling technique is given by E. N. Smith, H. M. Bozler, W. S. Truscott, R. C. Richardson, and D. M. Lee, in Proceedings of the Fourteenth International Conference on Low Temperature Physics, Otaniemi, Finland, 1975, edited by M. Krusius and M. Vuorio (North-Holland, Amsterdam. 1975).

${ }^{9} \mathrm{~A}$ small correction may be applied to the raw data to account for the unlocking of the normal fluid due to the rapidly decreasing viscosity below $T_{c}$. The magnitude of this correction in less than $0.3 \%$ in $\rho_{s} / \rho$ at our lowest temperatures.

${ }^{10}$ V. Ambegaokar, P. G. de Gennes, and D. Rainer, Phys. Rev. A $\underline{9}, 2676$ (1974).

${ }^{11} \mathrm{~V}$. Ambegaokar, in Lectures at Canadian Association of Physicists, International Summer School in Theoretical Physics. 1974 (unpublished).

${ }^{12}$ A. L. Fetter, Phys. Rev. B 14.2801 (1976), and other work to be published.

${ }^{13}$ Discontinuous changes in viscosity and superfluid density at the $A B$ phase change were first observed by Alvesalo et al. (Ref. 2). Further work is in progress on the analysis and interpretation of the viscosity data from the present experiment. The data do show the quantity $\left(\eta_{A}-\eta_{\boldsymbol{b}}\right) / \eta_{\boldsymbol{b}}$ to have a value of about 0.13 at $T_{A B}$.

${ }^{4}$ Y. Kuroda and A. D. S. Nagi, to be published.

\title{
New Applications of X-Ray Standing-Wave Fields to Solid State Physics
}

\author{
S. Kjaer Andersen,* J. A. Golovchenko, and G. Mair $\dagger$ \\ Institute of Physics, University of Aarhus, DK-8000 Aarhus C, Denmark
} (Received 11 August 1976)

\begin{abstract}
An x-ray standing-wave-field apparatus has been constructed that demonstrates an impurity atomic position resolution of $\sim 0.02 \AA$. The method is applied to the measurement of ion-implantation-induced lattice relaxation. It is pointed out that the method is also suited to determine surface impurity locations perpendicular to crystal surfaces.
\end{abstract}

It is by now well known that $\mathrm{x}$-ray standingwave fields are excited in nearly perfect crystals under the conditions of strong diffraction. The systematic study of this dynamical diffraction effect in the case of the Laue geometry has led to several interesting applications of $x$-ray diffraction to solid state and atomic physics via the Bormann effect, ${ }^{1}$ pendellösung fringe measurements, ${ }^{2}$ and $x$-ray interferometry. ${ }^{3}$ The Bragg geometry, despite its simpler nature, appears in recent times to have played a somewhat minor role in terms of dynamical diffraction applications. Nevertheless, using the recent development of solid-state $\mathrm{x}$-ray detectors of high-energy resolution, Golovchenko, Batterman, and Brown ${ }^{4}$ were able to demonstrate the possibility of using the peculiar properties of the Bragg geometry along with $\mathrm{x}$-ray fluorescence techniques to locate the atomic position of impurity atoms in a crystal.

This work, following the studies of Batterman, ${ }^{5,6}$ utilized the dynamical-theory prediction ${ }^{7}$ that for 
an incident beam direction near the Bragg condition a single standing-wave pattern (for each state of polarization) is excited in the crystal whose nodal planes lie parallel to and have the periodicity of the diffracting planes. Furthermore, as the incident glancing angle is scanned through the range of total reflection, the nodal plane will move from the point of maximum electronic charge density to the point of minimum charge density for that Fourier component of the charge density associated with the reflection considered. This standing-wave field was then used as a probe to excite impurity $\mathrm{x}$-ray fluorescence radiation, the angular yield of the $x$-ray fluorescence being proportional to the angular dependence of the standing-wave electric field intensity at the impurity location. Unfortunately, accompanying strong diffraction effects are strong angularly dependent extinction effects which generally result in a further and unwanted modulation of impurity angular fluorescent yields. It was an important aspect of the work of Ref. 4 that impurity atoms were located at depths much smaller than the minimum extinction length so that only variations in the impurity fluorescence yield due to standing-wave field motion were observed. Then it follows that from the angularfluorescence-yield curve for a given reflection, the atomic location of the impurity, relative to the associated electron-density Fourier component, can be determined. Knowledge of the phase of the structure factor, for the reflection of interest, locates the electron-density Fourier component relative to the crystal nuclei in the atomic planes, and therefore the atomic position of the impurity relative to the atomic planes is also determined. ${ }^{8}$ Thus, for the case studied in Ref. 4, the expected result of arsenic atoms lying in silicon (220) atomic planes was obtained.

During the past few years we have constructed an $\mathrm{x}$-ray diffraction facility at Aarhus University specifically for the type of measurement first mentioned above. One purpose has been to determine the kind of atomic-position resolution one may be able to achieve in such a measurement. (The actual position resolution of the measurement in Ref. 4 was quite poor.) During the course of this study a few further applications of the technique have become apparent. It is the purpose of this Letter to point out briefly the degree of resolution we have achieved and the new applications of the method in the areas of radiation damage and surface physics. While the work reported in this Letter in no way represents a surface exper- iment, we are especially interested in communicating the results to surface physicists. We hope to make it clear that the $\mathrm{x}$-ray standing-wave field lattice location technique applied to bulk impurities can also be utilized to locate impurity atoms on a crystal surface. In particular, the atomic position of an impurity perpendicular to the surface can be determined from the data by a simple analysis completely analogous to the one presented below. Equivalent accuracies of the order of $\sim 0.02 \AA$ should also be obtained in the surface-impurity case.

We therefore begin the discussion by presenting the predictions of the simple dynamical-diffraction, two-beam theory for the Bragg geometry.

Figure 1 shows the expected angular yield of fluorescence radiation from impurity atoms for several interplanar positions. The curves have been generated for Mo $K \alpha$ incident $\mathrm{x}$ rays on silicon (220) planes. The impurity is assumed to be so close to the surface (much closer than $1 \mu \mathrm{m}$ ) that extinction effects may be neglected. From these curves it may be seen that differences in position of a tenth of a planar spacing led to substantial modifications in the angular-yield curves. Thus with high angular resolution in the experimental angular scans and good counting statistics, position resolution for impurity atoms of about $1 \%$ of a planar spacing should be obtainable. The (220) reflectivity curve is also shown in the figure.

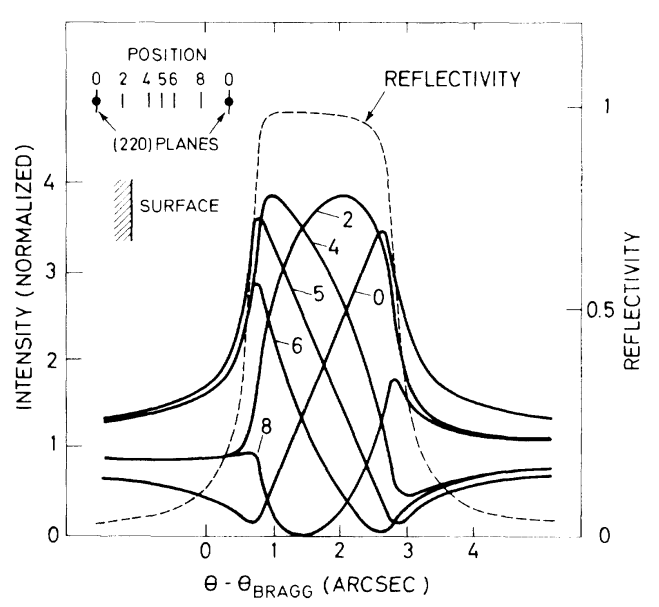

FIG. 1. Theoretical relectivity and electric-field strength versus incidence angle for several atomic positions between (220) planes. The integers labeling each curve give the atomic position in units of the planar spacing upon division by 10 . The curves are normalized to unity far off the Bragg condition. 
In order to achieve this goal, several modifications in the experimental apparatus described in Ref. 4 were required. While the details of the new system will be described elsewhere, we briefly mention here the two essential modifications. First, in order to obtain a highly collimated incident beam, an asymmetrically cut collimator crystal of the type described by Renninger ${ }^{9}$ was used. This resulted in an incident-beam collimation of $\sim \frac{1}{7}$ of the (220) natural reflection width. In order to obtain high counting statistics with a conventional $x$-ray source, it has been necessary to spend on the order of $10 \mathrm{~h}$ on an angular scan. Keeping angular stability commensurate with the angular resolution mentioned above over such a time period is a nontrivial problem. The second modification therefore consists of a piezoelectrically driven sample goniometer, servomechanically controlled from the Bragg reflection detector. With this system, angular drifts of $\$ 0.02 \mathrm{sec}$ over the course of a day have been achieved.

A polished silicon crystal with a surface cut parallel to the (220) planes was implanted at room temperature with $60-\mathrm{keV}$ arsenic ions. The total arsenic dose was $10^{15} / \mathrm{cm}^{2}$. The range of $60-\mathrm{keV}$ arsenic ions in silicon is roughly $400 \AA$ with a range straggling of $\sim 30 \%$. The sample was then annealed at $800^{\circ} \mathrm{C}$ for $\frac{1}{2} \mathrm{~h}$ to reduce or eliminate lattice damage caused by the implantation.

Figure 2 shows the Bragg reflection curve taken with this sample. It will be seen that an essentially perfect Darwin-Prins reflection curve is ob-

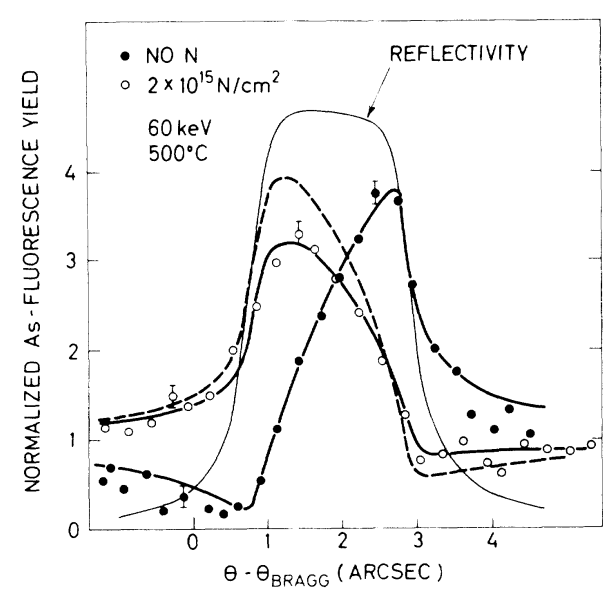

FIG。2. Experimental reflectivity and arsenic-fluorescencee-yield angular scans. The dashed curve indicates the nor malized y ield from the nonrandom distribution of arsenic atoms in the nitrogen-implanted sample. tained. Also shown in Fig. 2, in the filled circles, is the angular-yield curve for arsenic fluorescence $K \alpha$ radiation emanating from the sample. Comparison of these data points with the curves of Fig. 1 shows the arsenic atoms to be virtually substitutional.

During the course of our studies we were led to perform the following experiment. In a series of arsenic-doped silicon samples, prepared by the method described above, hot $\left(500^{\circ} \mathrm{C}\right)$ implantations of $60-\mathrm{keV}$ nitrogen ions were performed. The nitrogen dose ranged from zero to $5 \times 10^{15} /$ $\mathrm{cm}^{2}$ from the first to the sixth sample. Great care was taken to have a uniform nitrogen concentration across each sample. The samples were then inserted in the $x$-ray apparatus and the angular fluorescence yield of the arsenic atoms was observed.

The resulting angular-yield curves were transformed from the substitutional result to that corresponding to an increasing translation of arsenic atoms towards the surface with increasing dose. The reflection curves for all samples remained essentially perfect. A typical fluorescence scan is shown in the data, appearing as open circles in Fig. 2, which corresponds to a nitrogen dose of $2 \times 10^{15} / \mathrm{cm}^{2}$. Note that in going from zero nitrogen concentration to this dose, the maximum in the fluorescence yield has shifted from the high-angle to the low-angle side of the reflection curve. With increasing doses, the curves continue to shift until, at $5 \times 10^{15} / \mathrm{cm}^{2}$, the maximum again appears on the high-angle side of the reflection curve. Except for a small complication, to be discussed shortly, the nitrogen implantation procedure has allowed us to scan through the theoretical curves shown in Fig. 1.

The explanation for this curious effect, which so beautifully reproduces the range of results obtainable from an $\mathrm{x}$-ray location experiment, appears clear when it is noted that the range of 60$\mathrm{keV}$ nitrogen ions is $\sim 2000 \AA$ with a straggling of $\sim 50 \%$. Thus the nitrogen ions and their associated radiation damage lie beneath the arsenic atoms which are in an almost unperturbed top layer of silicon atoms. In the nitrogen layer below, there is a small lattice expansion that causes the outer layer to relax outwards, this outer layer still keeping the periodicity of the bulk. That is, all the arsenic atoms simultaneously shift outwards relative to the bulk. Because of the shallow depth of both of these layers, as compared with the minimum extinction length $(\sim 1$ $\mu \mathrm{m})$, the standing-wave field patterns will be vir- 
tually unaffected by nitrogen implantation. Thus relative to the standing-wave field patterns, and the bulk crystal which determines the phase of the former, all the arsenic atoms are shifted, with the shift proportional to the nitrogen dose.

We are thus provided simultaneously with a tool for measuring, on a microscopic scale, the integrated lattice relaxation of the nitrogen layer and with a means of verifying the dynamical-theory predictions of Fig. 1.

The lattice-relaxation data for different doses of nitrogen were fitted with the theory shown in Fig. 1, using a nonlinear least-squares program in order to obtain the actual lattice-relaxation values. The relaxation is clearly an expansion with the values shown in Fig. 3. We are not aware of any similar measurements of implantation-induced relaxation.

A small complication in the above analysis results from the fact that implantation past an initially substitutional layer of arsenic in silicon is known from channeling measurements to result in a sizeable fraction $(\sim 35 \%)$ of arsenic atoms to be displace from local substitutional sites. Attempts to determine the atomic location by channeling studies have not indicated any specific site for this nonsubstitutional fraction. Indeed, the assumption that this fraction is distributed randomly through the lattice (perhaps coupled to some complicated defect structure) is consistent with the channeling results. ${ }^{10}$ Similarly, in fitting the $\mathrm{x}$-ray data, a random component was determined from the assumption that only a locally substitutional and totally random distribution of arsenic atoms exists in the top layer. The resulting random fractions obtained generally agree

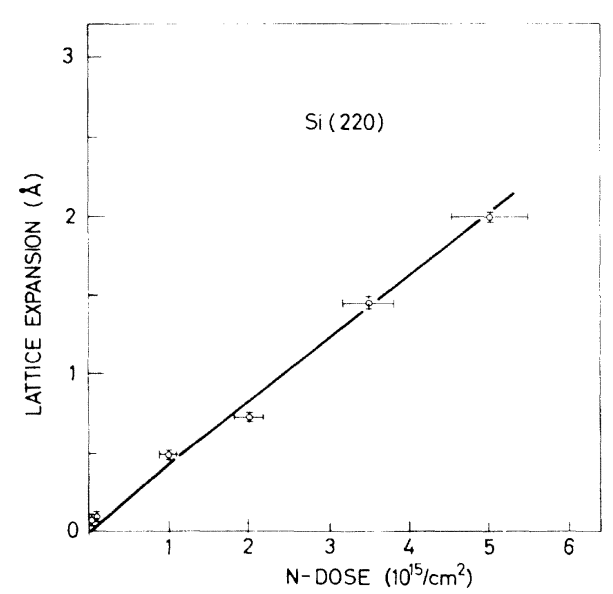

FIG. 3. Integrated lattice expansion of the nitrogen layer versus nitrogen dose. with the channeling measurement. The dotted curve in Fig. 2 shows the normalized yield curve for the nonrandom fraction of the arsenic atoms, which in this case was $64 \%$. The solid curves going through the data points in Fig. 2 are the fitted curves, including atomic positions relative to the bulk and random fraction.

The statistical accuracy of the relaxation measurement as deduced from the least-squares fitting program is $\sim \pm 0.02 \AA$. It is quite remarkable that position measurements with $0.71-\AA$ (Mo $K \alpha$ ) radiation can be achieved with such an accuracy.

While the implications of the data we have presented are clear for studies of lattice location and radiation damage in crystals, there is one application that perhaps transcends these. We note that the standing-wave fields we have been discussing exist not only inside the diffracting crystal, but also outside its surface to distances considerable greater than the planar spacing in the crystal. The standing-wave solution outside the surface may be looked upon as resulting simply from the interference between the incident and reflected beams. It will therefore be seen that all of the location discussion in Ref. 4 and in this brief note also applies to impurity atoms on the surface of the crystal. Since the diffracting planes of the sample crystal will normally be parallel to the surface, the location information will then be gathered perpendicular to the surface. Location information in this direction is difficult to obtain by low-energy electron-diffraction ${ }^{11}$ studies, and only recently with rather extended analysis has there been progress by this method. Note also that the number of impurity atoms used in this work corresponds approximately to one monolayer, if all the atoms were located at the surface.

It is therefore our hope that the work described above sufficiently illustrates the resolution, sensitivity, and simplicity of the standing-wavefield method to stimulate surface physicists to consider the implications $x$-ray analysis in their field.

The authors would like to express their gratitude to Ole Alstrup for preparing the samples used in this work and to Erik Uggerh $\varnothing \mathbf{j}$ for many fruitful discussions. This work has been supported by the Danish State Research Council and the Danish Council for Scientific and Technical Research.

\footnotetext{
*Present address: Department of Materials Science,
} 
Cornell University, Ithaca, N. Y. 14850 .

$\dagger$ Present address: Institut für Materialentwicklung, Kernforschungsanlage Jülich, D-517 Jülich, Germany. ${ }^{1}$ F. W. Young, Jr., T. O. Baldwin, and P. H. Dederichs, in Vacancies and Interstitials in Metals, edited by A. Seeger et al. (North Holland, Amsterdam, 1970) .

${ }^{2}$ P. J. E. Aldred and M. Hart, Proc. Roy. Soc. London, Ser. A 332, 223 (1973).

${ }^{3}$ U. Bonse and G. Materlik, Z. Phys. 253, 232 (1972).

${ }^{4} \mathrm{~J}$. A. Golovchenko, B. W. Batterman, and W. L.

Brown, Phys. Rev. B 10, 4239 (1974).

${ }^{5}$ B. W. Batterman, Phys. Rev. 133, A759 (1964).

${ }^{6}$ B. W. Batterman, Phys. Rev. Lett. 22, 703 (1969).

${ }^{7}$ M. V. Laue, Ann. Phys. (Leipzig) 23, 705 (1935).

${ }^{8}$ The inverse of this statement is also true. The fluorescence measurement locates the electron-density
Fourier component relative to the impurity. A previous knowledge of the impurity location in the lattice combined with the fluorescence measurement therefore determines the position (phase) of the Fourier component relative to the lattice. The knowledge of these phases for various Fourier components is required to experimentally reconstruct charge densities in the crystal. The phase of the Fourier component we use in this work is of course well known.

${ }^{9}$ M. Renninger, Z. Naturforsch. 16a, 1110 (1961)。

${ }^{10}$ L. Kalsen, thesis, Institute of Physics, Aarhus University, 1976 (unpublished).

${ }^{11} \mathrm{~T}$. N. Rodin and D. S. Y. Tong, Phys. Today 28 , No. 10, 23 (1975); T. N. Rodin and D. L。 Adams, in Treatise in Solid State Chemistry, edited by N. B. Hannay (Plenum, New York, 1976), Vol. 6, Chap. 5.

\title{
Raman-Active Lattice Vibrations of the Commensurate Superlattice in $2 \mathrm{H}-\mathrm{TaSe}_{2} \dagger$
}

\author{
John A. Holy, Miles V. Klein, W. L. McMillan, and S. F. Meyer* \\ Department of Physics and Materials Research Laboratory, University of Ilinois at Urbana-Champaign, \\ Urbana, Ilinois 61801 \\ (Received 26 July 1976)
}

\begin{abstract}
Raman spectra have been obtained on $2 \mathrm{H}-\mathrm{TaSe}_{2}$ at room temperature and in the commensurate charge-density-wave, superlattice state. At low temperatures six new low frequency modes are seen having symmetries consistent only with a symmetric, threecharge-density wave. A microscopic model of the lattice dynamics of this state is fitted to the four strongest new Raman lines. These are assigned to amplitude and phase modes of the charge-density wave.
\end{abstract}

In many layered transition-metal dichalcogenides an electronic instability produces a chargedensity wave (CDW), along with a periodic structural distortion (PSD) and a superlattice. ${ }^{1-3}$ It has previously been shown that the CDW's strongly affect the Raman spectra of these materials. ${ }^{4-6}$ This paper studies those new vibrations of the superlattice formed in $2 \mathrm{H}-\mathrm{TaSe}_{2}$ that couple most strongly to the $\mathrm{CDW}$. We first present Raman spectra from six new low-frequency zone-center phonons induced by the CDW. We then use these data with symmetry arguments to remove the ambiguities in the determination of the structure by electron ${ }^{1}$ and neutron ${ }^{2,3}$ diffraction. We finally present a microscopic model of the vibrations of the $\mathrm{Ta}$ ions about their new equilibrium sites.

The diffraction studies on $2 \mathrm{H}-\mathrm{TaSe}_{2}$ show a transition from a normal lattice to one with an incommensurate $\mathrm{CDW}$ at $122 \mathrm{~K}\left(T_{\mathrm{NI}}\right)$, followed at $90 \mathrm{~K}\left(T_{\mathrm{Cl}_{1}}\right)$ by a transition to a commensurate state. ${ }^{2}$ Fairly strong evidence exists for a $3 a_{0}$ $\times 3 a_{0} \times c_{0}$ unit cell in this state resulting from a superposition of three CDW's, but a single CDW with an $a_{0} \times 3 a_{0} \times c_{0}$ unit cell cannot be completely ruled out. Moncton has determined the ampli- tudes and relative phases of the atomic displacements in the commensurate state, but the results are insensitive to the absolute phase, ${ }^{3}$ which affects the point symmetry of the distorted structure and the Raman selection rules. We determine the phase from the observed selection rules and prove the existence of the three-CDW state.

Samples were grown by iodine-vapor transport. ${ }^{7}$ They had a $T_{\mathrm{NI}}$ of $122 \mathrm{~K}$ and a $T_{\mathrm{CI}}$ of $110 \mathrm{~K}^{8}$ The high value of $\boldsymbol{T}_{\mathrm{CI}}$ may be due to the low (30 ppm) level of transition-metal impurities. Raman measurements were made on a cleaved (0001) surface with use of a double grating monochromator equipped with a third monochromator and a 5145$\AA$ laser beam. The incident and scattered beams made external angles of $75^{\circ}$ and $0^{\circ}$ with the $c$ axis. Photon polarizations of hh (horizontal-horizontal) or vh (vertical-horizontal) relative to the scattering plane produced nearly pure $x x$ or $x y$ spectra. To verify assignments of symmetries, $x z$ spectra were observed in a vh experiment from a (1 100$)$ surface of a 1-mm-thick crystal. Cooling was provided by cold flowing helium gas. Temperatures were determined from the ratio of antiStokes to Stokes Raman intensities and are accu- 